\title{
REMOTE NETWORK LABORATORY TO SUPPORT MULTI-LEVEL NETWORK PRACTICE
}

\author{
Ninghan Zheng, Chengbin Quan, Xiaojun Wu, Shanshan Li, \\ Yongqiang Chen \\ Department of Computer Science and Technology, \\ Tsinghua University Beijing, 100084, China \\ zhengnh@tsinghua.edu.cn, quancbetsinghua.edu.cn, \\ xjwudtsinghua.edu.cn, \\ lishanshanetsinghua.edu.cn, chenyongqiangetsinghua. edu.cn
}

\begin{abstract}
The Computer Network Laboratory of Computer Science department in Tsinghua University provides experimental environment for undergraduate computer network serial courses offered by CS Dept. In order to meet the demand of different elective students, we combed experimental teaching system of computer network serial courses, then designed and implemented a remote network laboratory supported multi-level network practice. We mainly deployed three series of experimental system: Virtual Network Experiment System based on commercial network equipment, Network Protocols Experiment System named NetRiver and IPv4/IPv6 Transition Technologies Experiment System based on 4over6 tunneling technology. With the help of our network laboratory, the following goals can be achieved: 1) Remote. Students can access experimental environment from classroom, dormitory and etc.; 2) Multi-level. Experimental contents include both network principle and commercial realization, include both classical sliding window protocol, IPv4 or IPv6 data transceiver protocol, IPv4 or IPv6 data forwarding protocol, routing protocol and up-to-date IPv4/IPv6 Transition Technologies. Every network course can construct experimental content by combination of them; 3) Up-to-date. We introduced the latest technology in the field of computer network research, such as IPv6 network protocol and 4over6 tunneling technologies.
\end{abstract}

\section{KEYWORDS}

remote laboratory, laboratory education, experimental equipment, computer network

\section{INTRODUCTION}

The Computer Network Laboratory is an important part of the Computer Teaching Experiment Center in Computer Science department of Tsinghua University. The Lab provides experimental environment for undergraduate computer network serial courses offered by CS Dept. At present, the development of the laboratory mainly considers the following two aspects: Multi-level network practice and Innovative experimental method.

David C. Wyld et al. (Eds) : CSEN, SIPR, NCWC - 2016

pp. 39-53, 2016. @ CS \& IT-CSCP 2016

DOI : 10.5121/csit.2016.61004 
The consideration of the multi-level network practice comes from the fact that the lab serves different courses which have different target students and different teaching goals. We mainly divided the courses into three categories: Computer Network Curriculum as selective course for all the non-computer major students; Computer Network Principles Curriculum as compulsory course for the computer major students and Special Training in Computer Network Curriculum as limited optional course for the computer major students. The experimental teaching objectives of the three types of curriculums are different from each other, simultaneously they have common parts. So in order to achieve the goal of supporting different courses, we can deploy or implement serials of computer network experimental equipment and design variety of candidate experimental projects which can be effectively selected and combined.

Innovative experimental methods meet the demands of the tremendous development of electronic and computer techniques. The development of Internet technology, especially the development of the Cloud Computing technology, provides a strong support for the innovation of experimental methods. The experimental combination of the PCs and special experimental equipment in the past was gradually replaced by more flexible experimental methods. The remote experimental platforms based on $\mathrm{C} / \mathrm{S}$ mode or B/S mode are more popular with students. With the help of them, students can flexibly arrange experiment time and perform the experiment in classroom, dormitory, library and everywhere can access Internet.

Based on the above two points, we combed the knowledge of different computer network courses, designed and implemented three kinds of network experimental platforms to support multi-level network practice. These experimental platforms support remote experiment and be scalable to support the different number of elective students.

In this paper, we will introduce our attempt in trying to improve the computer network laboratory education in Computer Science department of Tsinghua University. We will first discuss the teaching system of computer network curriculums in section 2 secondly proposed our principles of designing computer network laboratory platforms in section 3, finally present the implementation of computer network laboratory education in Tsinghua University in section 4, and multi-level network practice supported by the principles will be presented simultaneously.

\section{TEACHING SYSTEM OF COMPUTER NETWORK EXPERIMENT}

Computer network experimental courses is not isolated, must be closely combined with the computer teaching curriculum system. Computer science emphasizes practice, so most of computer courses of CS Dept. in Tsinghua are provided with the corresponding experimental part. Figure 1. provides a brief description on the teaching system of computer experiment.

We used a tree-shaped model to describe the teaching system and divided computer experiment into five parts: Basic Experiment as the trunk which is the necessary prerequisite to other parts; four directions including Computer System Experiment, Software System Experiment, Computer Network Experiment and Application Technologies Experiment as leaves which represent the four secondary disciplines of CS and can be studied simultaneously. 


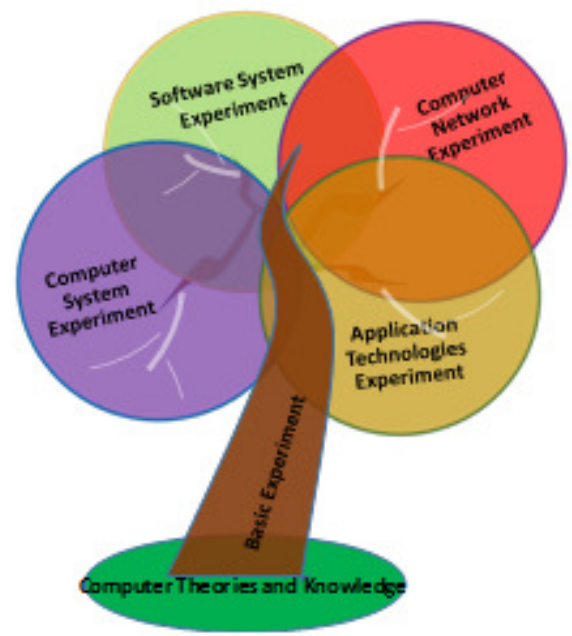

Figure 1. Teaching system of computer experiment

The Computer Network has been an independent secondary discipline of CS in 2015 in China because it plays a more and more important role in the economic development of our country. In the past we placed computer network courses into Application Technologies direction. Like MIT [1] and Stanford [2], we set the Computer Network Principles Curriculum as follow-up course of Computer Compose Principle Curriculum. Now we adjust them into parallel courses based on the following understanding:

- With the data structure knowledge and programming skills obtained in Basic Experiment, students can study network principles and protocols well, while Computer Compose Principle Curriculum focus on the principles of one local computer system include processor, memory, and etc.

- As an independent branch of teaching system, only a 48 hours required course is not sufficient. We added Special Training Serial Curriculums in which more deep practice is carried out as follow-up course of main curriculums in different directions such as Special Training in Operating System Curriculum followed Operating System Curriculum, Special Training in Compilation Principles Curriculum followed Compilation Principles Curriculum. Meanwhile we added Specialty Practice Serial Curriculums in junior summer semester, in which students complete a project with integrated knowledge of each direction. So Computer Compose Principle Curriculum belongs to Computer System Experiment and have its own subsequent Special Training Serial Curriculums and Specialty Practice Serial Curriculums.

Now we can draw up curriculum system of computer network experiment in Figure 2. In this figure, Computer Network Curriculum as selective course for all the non-computer major students is not drew up because of its separation from the teaching system of CS.

Computer Network Experiment spent 8 credit laboratory hours is a part of Computer Network Curriculum. This course is target at non-computer major students who are not expected to master thorough computer knowledge and programming skills. The curriculum teaches the principles of computer networks rather than network engineering or network buildup. So observation and 
analysis on network protocols rather than programming and implementation are expected in the experiment.

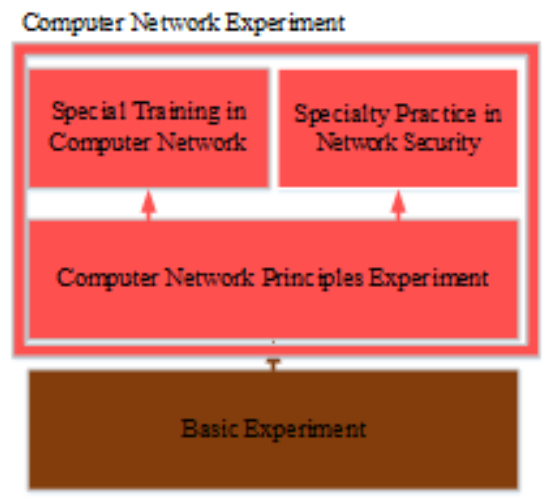

Figure 2. Curriculum system of computer network experiment

Computer Network Principles Experiment belong to Computer Network Principles Curriculum and worthy of 24 credit laboratory hours. It is a compulsory course for the computer major junior students in fall semester. The curriculum teaches the principles of computer networks with the classics textbook of "Computer Network" edited by Andrew [3]. Elective students with a strong foundation in computer programming are willing to comprehensively study the key network techniques and re-implement the important network protocols by hand.

Special Training in Computer Network followed Computer Network Principles Experiment is a full-time practical training course spend 48 hours. 24 credit laboratory hours spent in Computer Network Principles Experiment are not sufficient to do comprehensive practice in network protocols and cannot accommodate frontier network knowledge and technology. Special Training in Computer Network aims to be the appropriate successor.

Specialty Practice in Network Security is also a full-time practical training course. The 200 hours will be used to complete a project for Network Security. A set of special experimental equipment is dedicated to the course. This is beyond the scope of this paper.

\section{PRINCIPLES OF DESIGNING COMPUTER NETWORK LABORATORY PLATFORM}

In view of the discussion above, we propose several principles in designing computer network laboratory platforms (Figure 3.):

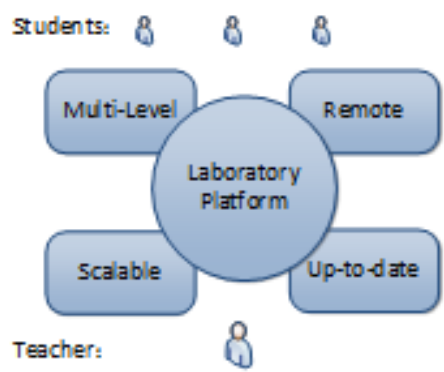

Figure 3. Principles in designing computer network laboratory platform 
- Multi-level

We dedicate to design laboratory platform for three main courses: Computer Network Experiment, Computer Network Principles Experiment and Special Training in Computer Network. Computer Network Experiment pay attention to observation and analysis on network protocols. Computer Network Principles Experiment encourage reimplementation the important network protocols. Specialty Practice in Network Security continue to strengthen the former mission and introduce new network technology.

\section{- Remote}

Internet technology, especially Cloud Computing technology, create possibilities for remote experiment and cloud-based experiment [9-11]. Students can flexibly arrange experiment time and perform the experiment in classroom, dormitory, library and anywhere can access Internet. We wouldn't consider software-based virtual network devices like $[9,10]$ because of their unreal results. We'd like to publish real physical network devices to students in Internet, thus they can interact with real equipment.

- Scalable

Capacity of experiment course, especially the elective courses, fluctuates wildly from year to year. The laboratory platforms should be scalable according to student number. As remote experiment resource according to the planning requirements of the last section, it should be easier to be deployed and released.

- Up-to-date

Network technology have been developing rapidly and new technologies continue to appear. Students like to practice on rich and up-to-date network technologies including IPv6 network protocol, IPv4/IPv6 Transition Technologies and wireless network technology. In the original laboratory, these experimental contents could not be supported. So we must continuously design and implement new laboratory platforms to keep pace with the times.

In the past work, there are many kinds of experimental methods:

- Commercial network equipment: Students are curious of how the real network instruments including switches and routers work. Although network buildup which should be included in engineering elective course is not the focal point of our experiment, interacting with them we can analysis the work principles of network protocols through packet capture software.

- Visual learning tools: The tools use images or Flash to display the results of interaction with the simulated network device $[4,5]$. They are often used to rapidly verify the correctness of operating and configuration on device and are suitable for novice learning such as Cisco Networking Academy [6]. But we cannot capture the intermediate results of interaction, and then the protocol analysis cannot be carried out.

- Network programing: Operating system provides API for user-end network programing such as Winsock and Linux socket. But the core network protocol stacks are encapsulated in 
system modules and transparent to user. Students are difficult to operate the network protocols.

- Network simulation tools: There are so many tools of this type: OPNET, J-Sim, NS-2, NS-3, KivaNS and etc. They play a great role in scientific research. But we think they are not suitable to undergraduate experimental teaching no matter use them directly [7] or wrap them with a friendly front end [8]. These tools are not intuitive and require deep knowledge and experience. Students have to spend a lot of time to learn the manuals.

- Virtual machines: Use VMs, we can do network experiment remotely [9, 10]. But also we need guest OS to provide software-based virtual router, firewall and VPN.

\section{IMPLEMENTATION OF COMPUTER NETWORK LABORATORY EDUCATION}

\subsection{Computer Network Laboratory Equipment}

Based on the above discussion, we propose three categories of experimental methods to support multi-level experiment:

\subsubsection{Virtual Network Experiment System}

We deployed cloud-based virtual network experimental platform of Ruijie Corporation [13] to support remote experimental environment based the real network devices. Figure 4. depicts one experimental group with Virtual Network Experiment System and now we deployed 8 groups. Each group consists of a set of real physical network devices include one router, one layer 3 switch and one wireless AP.

Cloud-based virtual network experimental system is a special industrial control computer using virtualization technology which can simultaneously load multiple VM images running Windows or Linux. There are 10 physical connection channels between the system and layer 3 switch. So it can simulate 10 PCs.

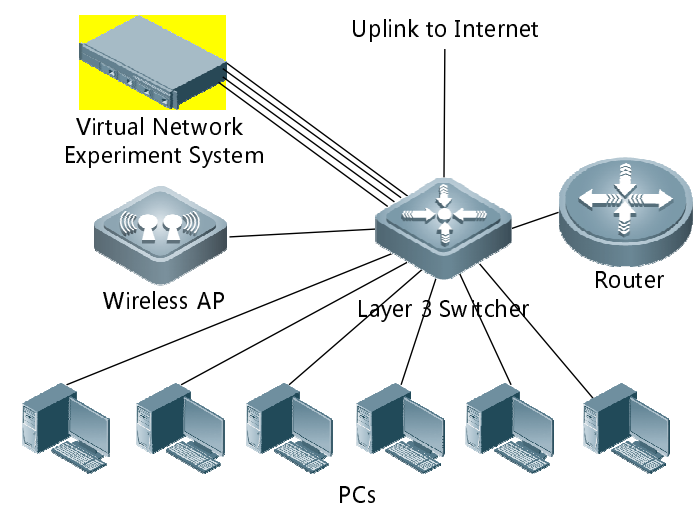

Figure 4. A experimental group with Virtual Network Experiment System 
There are two main ways to do experiment: The first way is using PC to do the traditional local experiment. The other way is using virtual terminals produced by virtual network experimental system to do remote experiment. Students remotely access Virtual Network Experiment System through browser and log in the VM OS generated by system. For students, it seems that the real network devices are around them now.

It seems strange in Fig. 4. that the router is out of proper place. In a classic network topology, the router should be laid between the uplink and switch. But when students do router experiment remotely, they will probably lose the link because the router not retain it. So our solution is using the lookback interface as experimental interface instead of uplink interface when do the router experiment.

With the help of packet capture software like WireShark [14], students can observe and analysis explicit working process of the network protocols in our experimental environment. A group package analysis based experiment supported are list in Table 1:

Table 1. Experiment supported by virtual network experiment system

\begin{tabular}{|l|l|l|}
\hline Tag & Experiment Name & Experiment Object \\
\hline 111 & FAT AP with single SSID & $\begin{array}{l}\text { Configuration on wireless AP with single SSID in FAT } \\
\text { mode }\end{array}$ \\
\hline 112 & FAT AP with multiple SSID & $\begin{array}{l}\text { Configuration on wireless AP with multiple SSID in } \\
\text { FAT mode }\end{array}$ \\
\hline 113 & AP bridge in one vlan & Bridge the same vlan using two APs \\
\hline 114 & AP bridge in different vlans & Bridge different vlans using two APs \\
\hline 121 & ARP protocol & Analyse principle of ARP protocol \\
\hline 122 & 802.3 package & Analyse package of 802.3 \\
\hline 123 & 802.1 package & Analyse package of 802.1Q \\
\hline 124 & STP BPDU & Analyse package of Spanning Tree protocol \\
\hline 125 & MSTP BPDU & Analyse package of Multiple Spanning Tree protocol \\
\hline 126 & Loop effect & Analyse Loop effect without Spanning Tree protocol \\
\hline 127 & LLC forward & Analyse package forwarding of ARP protocol \\
\hline 131 & Static route & Config and Analyse static route \\
\hline 132 & RIP & Config and Analyse RIP protocol \\
\hline 133 & OSPF & Config and Analyse OSPF protocol \\
\hline 141 & TCP & Analyse TCP protocol \\
\hline 142 & UDP & Analyse UDP protocol \\
\hline 151 & DHCP & Config and Analyse DHCP protocol \\
\hline 152 & DHCP relay & Config and Analyse DHCP relay protocol \\
\hline 153 & FTP & Config and Analyse FTP protocol \\
\hline 154 & HTTP & Config and Analyse HTTP protocol \\
\hline & &
\end{tabular}


"Tag" in this table plays a role in category: The first number distinguishes the platforms: " 1 " for Virtual Network Experiment System, "2" for Network Protocols Experiment System and "3" for IPv4/IPv6 Transition Technologies Experiment System; the second represents the layer of TCP/IP 5-layer network protocols, for example "2" means data link layer; the last one is sequence number of the same layer experiment in one system.

The pros and cons of the system include: 1) It suits the non-computer major students without strong programming skills to study basic network principles. And also can be a foundation correlation method to the majors when they realization network protocols by programing; 2) It creates possibilities for remote access to real physical network equipment instead of softwarebased virtual device. Meanwhile traditional local experimental environment reserved; 3) It is scalable by adding the number of groups; 4) With the help of Ruijie wireless AP and 4G switch, wireless experiment is added to the laboratory.

\subsubsection{Network Protocols Experiment System (NetRiver)}

We designed and implemented the Network Protocols Experiment System named NetRiver to support programing experiment. Early version named NetRiver 2000 was released in 2007 [12]. New version of NetRiver 3000 was released in 2015. The topology is depicted in Figure 5.

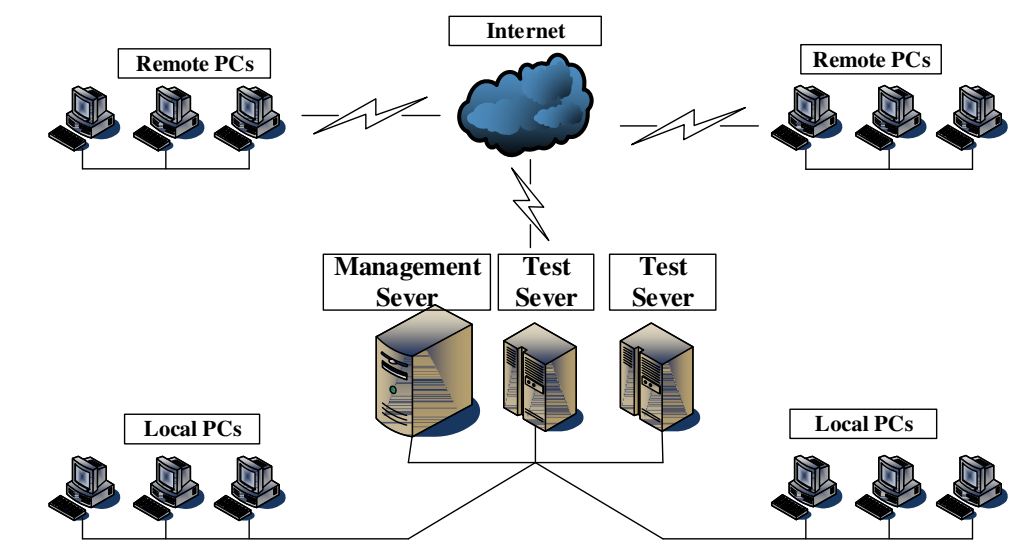

Figure 5. A experimental group with Virtual Network Experiment System

The system consists of a management server, variable number of test server and a user-friendly integrated development software client.

Management server is used to manage the students' information, experiment content. The results of experiment can be submitted to the server, then the statistical results are generated automatically for instructors. The visual client provides edit, compile, execute and debug environment. Test server acts as the key of the system. The TCP/IP 5-layer network protocols have been realized in it, meantime a serial of APIs are open to students. Students can conveniently focus on specified segments of these network protocols according to experimental requirements, without concerning about the context. After that students can test their tasks with the test server, contrary to which, in a real network environment this type of experimental data packages will be discard as error data. 
Students can edit, compile, execute and debug offline in any PC client. They realize the experiment code of network protocol segment, call the APIs provided by the system to constitute the complete protocol stack. Now they can login the management server and get the allocated test server. Test server will verify the task program by interacting with client.

This system uses scalable script description to define experiment content. Teachers can easily add new experiment content to it. Now the network protocols programing-based experiment are list in Table 2.

Table 2. Experiment supported by virtual network experiment system

\begin{tabular}{|l|l|l|}
\hline Tag & Experiment Name & Experiment Object \\
\hline 221 & $\begin{array}{l}\text { Sliding window } \\
\text { protocol }\end{array}$ & Realize sliding window protocol in Andrew textbook \\
\hline 231 & IPv4 sending and receiving & $\begin{array}{l}\text { Achieve the function of IPv4 Protocol in host protocol } \\
\text { stack }\end{array}$ \\
\hline 232 & IPv6 sending and receiving & $\begin{array}{l}\text { Achieve the function of IPv6 Protocol in host protocol } \\
\text { stack }\end{array}$ \\
\hline 233 & IPv4 transmission experiment & Achieve the function of IPv4 in router protocol stack \\
\hline 234 & IPv6 transmission experiment & Achieve the function of IPv6 in router protocol stack \\
\hline 235 & RIP protocol & Achieve RIP protocol in router protocol stack \\
\hline 236 & Protocol state machine & Achieve state machine in BGP protocol \\
\hline 237 & IPSec & Achieve IPSec protocol \\
\hline 241 & TCP & $\begin{array}{l}\text { Achieve encapsulation and sending, receiving of TCP, } \\
\text { analyzing process of TCP message }\end{array}$ \\
\hline
\end{tabular}

The pros and cons of the system include: 1) It is a protocols programing-based system. It helps students to study key network technologies which are hidden in system core of host OS or network devices' OS and difficult to practice. So it can be the key chain of practice for network direction computer major undergraduates; 2) Experimental resources of the system is being released online, students can finish the job remotely; 3) The main performance bottleneck locate in test server. But the number of test servers can also be increased by register in management server. 4) The achievement comes from IPv6 core router finished by Tsinghua, so it maintains the advanced nature of the technology.

\subsubsection{IPv4/IPv6 Transition Technologies Experiment System}

We developed new experiment system because IPv4/IPv6 Transition Technologies have emerged as hot researches. The work principles of IPv4/IPv6 Transition are also hidden in OS of hosts and network device. Some configuration-based experiment can be carried out with real commercial equipment. But programing-based experiment are seldom touched. Figure 6. describes process of the new system.

Main-body text is to written in fully (left and right) justified 11 pt. Times New Roman font with a 6pt. (paragraph) line spacing following the last line of each paragraph, but a 12pt. (paragraph) line spacing following the last paragraph. Do not indent paragraphs. 


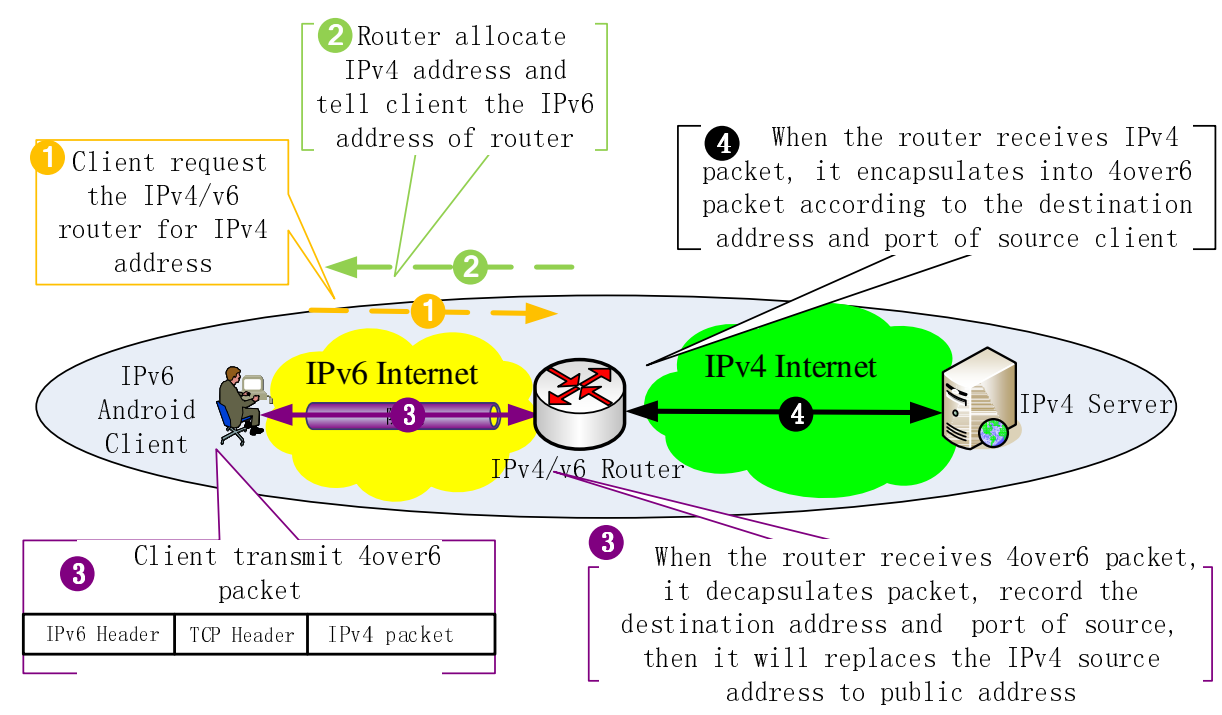

Figure 6. IPv4/IPv6 Transition Technologies Experiment System

In this scene, a user who has only IPv6 Internet wants to access the IPv4 server in IPv4 Internet. The work-flow will be:

- Client request the IPv4/v6 router for IPv4 address by broadcasting.

- Router responds to the request and allocate IPv4 address to client and tell client the IPv6 address of router.

- Client transmit 4over6 packet.

- When the router receives 4over6 packet, it decapsulates this packet, records the destination address and port of source, then it will replaces the IPv4 source address to public IPv4 Internet address.

- When the router receives IPv4 packet from IPv4 server, it encapsulates into 4over6 packet according to record of the destination address and port of client.

Now we have two parts to work at: IPv6 client and IPv4/IPv6 router. We call the IPv6 client as client of IPv4/IPv6 Transition Technologies Experiment System, IPv4/IPv6 router as the server.

In order to supplement the Android programing skill of undergraduates who have seldom practice on it in other courses. We deliberately user an Android mobile device as the client that a PC is also ok.

So two important experimental projects come out from Figure 6 (List in Table 3):

In the last semester, we only let the students to realize the client. The server was made into an Industrial Personal Computer which is more stable and reliable and deployed in campus network of Tsinghua. So the students can test their client remotely with the server. 
Table 3. Experiment supported by ipv4/v6 transition technologies experiment system

\begin{tabular}{|l|l|l|}
\hline Tag & Experiment Name & Experiment Object \\
\hline 331 & Client implementation & $\begin{array}{l}\text { Design and Implementation of client of IPv4/IPv6 } \\
\text { Transition Technologies Experiment System }\end{array}$ \\
\hline 332 & $\begin{array}{l}\text { Sever } \\
\text { implementation }\end{array}$ & $\begin{array}{l}\text { Design and Implementation server of IPv4/IPv6 } \\
\text { Transition Technologies Experiment System }\end{array}$ \\
\hline
\end{tabular}

The client running in Android OS can be divided into two parts: front-end and back-end.

Front-end is the UI programing in JAVA. The main functions include:

- Monitor the network and get the IPV6 address of the uplink physical interface.

- Start the back-end.

- $\quad$ Set the timer and refresh UI.

- Show the network information.

- Star the VPN service of Android OS.

Back-end programing in c interacts with the server. The main functions include:

- Access the server.

- Get the IPV4 address of the downlink virtual interface.

- $\mathrm{Read} /$ write virtual interface.

- Decapsulate IP packets.

- Communicate with the server by IPv6 socket

- Send keeplive message to the server

The server can also be deployed in Linux system. The main functions include:

- Create IPV6 socket, listen to the communication between the client and IPv4 server.

- Maintain the virtual interface, implement I/O on virtual interface.

- Maintain IPV4 address pool, allocate address to new client.

- Maintain the information table of clients, save the mapping relations between IPv4 address and IPv6 socket. 
- Read the data in IPV6 socket from client, handle the control message.

- Implement decapsulation function to data received by IPV6 socket, then write them to the virtual interface.

- Implement encapsulation function to data received by virtual interface, then send them to client.

- Listen to clients and send keeplive message.

The pros and cons of the system include: 1) The programing-based practice on IPv4/IPv6 transition technologies give a great challenge to computer major students. It can be good start to be network professionals; 2) The server of the system can be deployed in Internet, students can implement the client locally and test it with the server remotely; 3 ) It is very easy to add the new server if the server resource is insufficient; 4) IPv4/IPv6 transition technologies are new and popular.

\subsection{Computer Network Laboratory Curriculum}

With the support of laboratory equipment above, we can arrange the proper combination of experimental contents for the three Computer Network Laboratory Curriculum. Table 5 list the experimental contents in the latest semester.

Table 5. Experimental arrangement for computer network laboratory curriculum

\begin{tabular}{|c|c|c|c|c|c|c|}
\hline & \multicolumn{2}{|c|}{$\begin{array}{l}\text { Computer Network } \\
\text { Experiment }\end{array}$} & \multicolumn{2}{|c|}{$\begin{array}{l}\text { Computer Network } \\
\text { Principles Experiment }\end{array}$} & \multicolumn{2}{|c|}{$\begin{array}{l}\text { Special Training in } \\
\text { Computer Network }\end{array}$} \\
\hline Layer & Tag & Time (hour) & Tag & Time (hour) & Tag & Time (hour) \\
\hline \multirow{2}{*}{1} & 111 & 1 & & & 111 & 1 \\
\hline & 114 & 1 & & & 114 & 1 \\
\hline \multirow{2}{*}{2} & 126 & 0.5 & 221 & 8 & & \\
\hline & 127 & 0.5 & & & & \\
\hline \multirow{5}{*}{3} & 132 & 1 & 232 & 8 & 132 & 1 \\
\hline & 133 & 1 & 233 & 8 & 133 & 1 \\
\hline & & & & & 234 & 8 \\
\hline & & & & & 237 & 8 \\
\hline & & & & & 331 & 28 \\
\hline \multirow{2}{*}{4} & 141 & 0.5 & & & & \\
\hline & 142 & 0.5 & & & & \\
\hline \multirow{2}{*}{5} & 151 & 1 & & & & \\
\hline & 153 & 1 & & & & \\
\hline Amount & & 8 & & 24 & & 48 \\
\hline
\end{tabular}


Computer Network Experiment face the non-computer major students. Package analysis based experiment are more suitable. So they only get in touch with Virtual Network Experiment System. The experimental project covers all layers of the network protocols, and as many kinds of real network device as possible are introduced to students.

Computer Network Principles Experiment focus on working principles of network protocols. As a computer major student, he'd better to grasp the essentials of relative theoretic curriculum. Programing-based experiment are more suitable. Network Protocols Experiment System (NetRiver) is the best choice. But because of the limited time, only the most critical experimental contents are required.

Special Training in Computer Network is the continuation of Computer Network Principles Experiment. Two aspects are taken into account: More challenging tasks and neglected basic operation skills. For the former, IPv4/IPv6 transition technologies experiment which will take at least 20 hours for a well-skilled student are introduced. For the latter, small-scale network buildup experiment and package analysis based experiment are required. This is a result of feedback analysis on past students. Quite a few computer major students don't know how to build up a LAN when they go to work. Some of them cannot distinguish wireless AP from wireless router.

More experimental projects are supported by the three major system. It gives us the opportunity to alternate experimental contents every year. The projects list in TABLE IV can be replaced by equivalent ones.

\begin{tabular}{|c|c|c|c|}
\hline Text & Alingnment & Font & Followed by: \\
\hline Title & Centre & 20 pt. TNR, bold, small-caps & 24 pt. line sp. \\
\hline Authors & Centre & 13 pt. TNR & 12 pt. line sp. \\
\hline Addresses & Centre & 12 pt. TNR & \\
\hline emails & Centre & 11 pt. italic TNR & 18 pt. line sp. (last) \\
\hline Abstract heading & Left & 13 pt. bold italic TNR, small caps & 6 pt. line sp. \\
\hline Abstract text & Left & 10 pt. italic TNR & 12 pt. line sp. \\
\hline Keywords heading & Left & 13 pt. bold italic TNR, small caps & 6 pt. line sp. \\
\hline Keywords & Left, left, & 10 pt. italic TNR & $18 \mathrm{pt}$ line sp. \\
\hline Section headings & Left & 14 pt. bold TNR, small caps & 6 pt. line sp. \\
\hline Sub-section heads & Left & 12 pt. bold TNR & 6 pt. line sp. \\
\hline Sub-sub-sections & Left & 11 pt. bold TNR & 6 pt. line sp. \\
\hline Body text & Full (left/right) & 11 pt. TNR & $12 \mathrm{pt}$ line sp. (last) \\
\hline Figures & Centre & & 6 pt. line sp. \\
\hline Figure captions & Centre & 11 pt. TNR & 12 pt. line sp. \\
\hline References & Left & 10 pt. TNR (as shown) & 6 pt. line $\mathrm{sp}$ \\
\hline
\end{tabular}

\section{CONCLUSIONS}

According to the teaching system of computer network experiment in CS Dept. Tsinghua University, we proposed the principles of designing computer network laboratory platform: multi-lever, remote, scalable and up-to-date. Then we design and implement three types of network experimental equipment: Virtual Network Experiment System based on commercial network equipment, Network Protocols Experiment System named NetRiver and IPv4/IPv6 Transition Technologies Experiment System based on 4over6 tunneling technology. These devices satisfy our designing principles. By proper combination of the experimental contents 
supported by them, we set up computer network laboratory education for the three experiment courses: Computer Network Experiment for non-computer major students, Computer Network Principles Experiment for computer major students and Specialty Practice in Network Security for network professional students.

\section{REFERENCES}

[1] MIT., Computer System Engineering, Spring 2016, http://web.mit.edu/6.033/www/

[2] Stanford University, Introduction to Computer Networking, Autumn 2011, http://www.scs.stanford.edu/11au-cs144/

[3] Andrew S. Tanenbaum, 2004, "Computer network", The 4th edition, Beijing: Tsinghua University Press

[4] S. Wei, Z. Qunyi,L. Xuefen, "Research of Educational Technology of Computer Networks", 2010 International Conference on E-Health Networking, Digital Ecosystems and Technologies, vol.4 pp. 192-195, 17-18 April 2010

[5] J. Janitor, F. Jakab, K. Kniewald, "Visual Learning Tools for Teaching/Learning Computer Networks", ICNS '10 Proceedings of the 2010 Sixth International Conference on Networking and Services, Pages 351-355

[6] Cisco Networking Academy, http://www.cisco.com/go/netacad

[7] N. Al-Holou, K. K. Booth, E. Yaprak, "Using computer network simulation tools as supplements to computer network curriculum", Frontiers in Education Conference, 2000. FIE 2000. 30th Annual, S2C/13 - S2C/16 vol.2, 18-21 Oct 2000

[8] N. Jovanovic, Z. Jovanovic, et al., " Computer Network Simulation and Visualization Tool for Educational Purpose", 11th International Conference on Telecommunication in Modern Satellite, Cable and Broadcasting Services (TELSIKS), 2013, vol.4 pp. 579-582, 16-19 Oct 2013

[9] M. W. Bazzaza, K. Salah,"Using the Cloud to Teach Computer Networks", 2015 IEEE/ACM 8th International Conference on Utility and Cloud Computing (UCC),pp. 310-314, 2015

[10] C. Yan, "Bulid A Laboratory Cloud for Computer Network Education", 2011 6th International Conference on Computer Science \& Education (ICCSE), pp. 1013-1018, 2011

[11] L. Bellido, V. Mateos, et al., "Remote Access to Computer Networking Laboratories", 2012 9th International Conference on Remote Engineering and Virtual Instrumentation (REV), pp. 1-6, 2012

[12] Xu M., XU K., et al., "NetRiver, a computer network experiment system", SCE '08: Proceedings of the 1st ACM Summit on Computing Education in China on First ACM Summit on Computing Education in China,October 2008

[13] Ruijie Corporation, http://www.ruijie.com.cn/

[14] WireShark software, https://www.wireshark.org/ 


\section{AUTHORS}

Ninghan Zheng: Born in 1979. Master Degree of CS. Work in Department of Computer

Science and Technology, Tsinghua University in Beijing, China. Engaged in experimental teaching research of Computer Network and Embedded System.

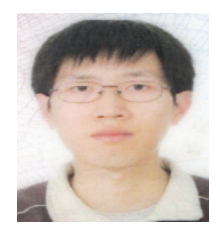

